\title{
Absence of Consistent Pattern Between Seasons or Among Species in Effects of Leaf Size on Insect Herbivory
}

\section{Xiang Zhang}

Anhui University

\section{Li Feng}

Anhui University

Zhiyun Lu

Xishuangbanna Tropical Botanical Garden

Bo Wang ( $\nabla$ yangblue@ahu.edu.cn )

Anhui University https://orcid.org/0000-0003-1010-6573

\section{Research Article}

Keywords: biotic interaction, herbivore, leaf area, non-monotonic, plant fitness

Posted Date: December 14th, 2021

DOI: https://doi.org/10.21203/rs.3.rs-1141805/v1

License: (c) (i) This work is licensed under a Creative Commons Attribution 4.0 International License. Read Full License 


\section{Abstract}

Insect herbivory on plant leaves is a major determinant of plant fitness, especially the growth and survival of tree seedlings in forests. Leaf size is believed to significantly affect the intensity of herbivory. Studies often assume the relationship between leaf size and herbivory to be monotonic; however, it is influenced by many factors-the magnitude and direction of whose effects are different-indicating a complex nonmonotonic pattern. In this study, we investigated the herbivory of 5754 leaves of 422 seedlings belonging to 43 subtropical tree species over two seasons in southwest China. The effects of leaf size on herbivory differed among seasons; a hump-shaped pattern was detected in December, while a pattern of monotonic increase was detected in September. A variety of patterns including complex non-monotonic patterns presenting U-shaped and hump-shaped patterns, as well as patterns indicating monotonic decrease and increase existed, although most species displayed a leaf-size-independent pattern. The relationship between leaf size and insect herbivory does not follow a constant rule, but differs across species and seasons, indicating that the effects of leaf size on the foraging preferences of insect herbivores may be contingent on both external (e.g., temperature) and intrinsic (e.g., other leaf traits) factors. Therefore, a one-off survey focusing a few species may provide misleading understanding on the overall pattern of the effect of leaf size on herbivory. Similar variations may also exist in other ecological processes, which should be given due consideration in future studies on biotic interactions.

\section{Introduction}

Leaf size varies dramatically both across and within species, and even in the same individual plant (Poorter and Rozendaal 2008; Hulshof and Swenson 2010; Diaz et al. 2016; Zhang et al. 2016; Wright et al. 2017). Many studies have discussed the potential explanations or reasons for such large variations in leaf size, for example, precipitation, moisture, temperature, irradiance, soil fertility, genotype, and crown position (McDonald et al. 2003; Hulshof and Swenson 2010; Wright et al. 2017; Eisenring et al. 2021). Consequently, the variation in leaf size has significant ecological implications, and affects many ecological processes including leaf expansion time, photosynthesis, transpiration rate, thermal regulation, and self-shading (Moles and Westoby 2000; Falster and Westoby 2003; Yates et al. 2010; Leigh et al. 2017; Hu et al. 2020).

Insect herbivory (hereafter referred to as herbivory) is a major factor that results in leaf damage and death, and can in turn affect plant fitness, especially the growth and survival of tree seedlings in forests (Schowalter et al. 1986; Eichhorn et al. 2010; Barton and Hanley 2013; Jactel et al. 2021). Leaf size is believed to significantly affect the intensity of herbivory (Brown and Lawton 1991; Moles and Westoby 2000; Cardenas et al. 2014; Li et al. 2021). Several potential explanations for this have been presented: 1) logically, a larger leaf usually holds more biomass and represents more absolute nutrients, and also provides the insect herbivores (also referred to as herbivores in this article) better shelter because of their larger area; 2) a larger leaf often requires a longer expansion time, which is the period when the leaf is most vulnerable to herbivory (Moles and Westoby 2000);3) larger leaves may be easier to detect than smaller ones (the apparency theory, Feeny 1976). Therefore, larger leaves may be expected to endure 
heavier herbivory. Furthermore, leaf size is often correlated with leaf age, and indirectly affects the foraging preferences of insect herbivores (Coley 1980; Aide 1993; Low et al. 2009; Moreira et al. 2016); for example, young shoot tips are more vulnerable to herbivory than base leaves. Therefore, interspecific differences in the correlation between leaf size and leaf age may lead to differences in leaf size preference of insect herbivores; for example, insect herbivores prefer small leaves of Betula pubescens, but larger leaves of Salix phylicifolia (Bogacheva 1994). Furthermore, leaf size is also related to many other leaf traits such as specific leaf area, chemical and physical defenses, and nutrient content, all of which can influence the leaf size preference of herbivores (Ackerly et al. 2002; Cardenas et al. 2014; Zava and Cianciaruso 2014).

Most studies assume a monotonic trend in the relationship between leaf size and herbivory (Bogacheva 1994; Moles and Westoby 2000; Cardenas et al. 2014; Li et al. 2021). However, as discussed above, the relationship between leaf size and herbivory is affected by many factors, and the magnitude and direction of the effects differ among factors, indicating that the relationship between leaf size and herbivory may show a complex non-monotonic pattern that has been found in many other ecological processes (Elliott and Irwin 2009; Yan and Zhang 2014; Thein et al. 2021; Zhang et al. 2021). Current evidence seems to support the above hypothesis that herbivory can be positively or negatively related to, or be independent of leaf size (Bogacheva 1994, 2002; Moles and Westoby 2000; Potter et al. 2012; Cardenas et al. 2014).

In order to gain a more comprehensive understanding of how leaf size affects insect herbivory, we investigated the herbivory of 5754 leaves of 422 seedlings belonging to 43 tree species in a subtropical evergreen broad-leaved forest for two seasons. We aim to address the following three questions: 1) Does a nonmonotonic pattern exist in the relationship between leaf size and insect herbivory across plant species? 2) Does leaf size affect insect foraging preferences within each individual species; if so, is there a fixed rule deciding the preferences? 3) Do the two above-mentioned predictions show similar patterns across seasons?

\section{Material And Methods}

\section{Study site}

This study was conducted in a subtropical forest in the Ailao Mountains, in southwest China $\left(24^{\circ} 32^{\prime} \mathrm{N}\right.$, $101^{\circ} 01^{\prime} \mathrm{E}$, altitude $\sim 2000 \mathrm{~m}$ ). The dominant species are Lithocarpus hancei, Lithocarpus xylocarpus, and Castanopsis wattii, etc. In January 2015, a heavy snowfall caused extreme damage to the forest, with approximately $50 \%$ of tree canopies being destroyed. In April 2015, we initiated a long-term project to monitor seedling regeneration after the snow damage. In total, 388 permanent sample plots $(1 \mathrm{~m} \in 1 \mathrm{~m})$ were established in the forest to monitor seedling survival dynamics.

\section{Experimental design}


This study was conducted in September and December of 2020. We randomly selected 30 and 45 plots in September and December, respectively, with 15 plots common for both months. In each survey, we identified all seedlings of woody species in each plot. For individuals with no more than 30 leaves, we measured the leaf size and herbivory of each leaf; for individuals with a large number of leaves, 30 leaves were randomly selected. For species with compound leaves, each leaflet was treated as a measurement unit.

The length and width of each leaf were measured, and the leaf size was calculated using the ellipse area formula. For a few leaves that could not be measured due to excessive feeding during the investigation, we measured the size of the leaf nearest to the leaf under focus, instead. Leaf damage was defined as the proportion of leaf area consumed by leaf chewers, which were the dominant insect herbivores in the forest under our study. Other herbivores such as piercing and sucking insects were not considered because 1) piercing/sucking damage was rarely found during our field survey, and 2) it was difficult to measure the piercing/sucking damage using the method we used to determine damage from chewing. The damage to all the leaves was visually estimated by a single investigator, to reduce errors caused by the introduction of subjectivity when different observers are involved; the proportions of leaf damage visually estimated by the investigator and those accurately measured using the scanner showed good correlation (Pearson correlation coefficient, $r=0.877, P<0.001, n=60$ ).

\section{Data analysis}

All analyses were performed using $\mathrm{R}$ (version 4.0.5). We analyzed the effects of leaf size, both across and within species. Phylogenetic generalized least squares regression was used to quantify the effects of leaf size on herbivory across species (function 'pgls' in package 'caper'). Three indicators of herbivory were tested: 1) herbivory frequency, that is, the ratio of the number of leaves damaged by herbivores to the total number of leaves of a specific species sampled; 2) the arithmetic average herbivory severity, that is, the mean value for all the sampled leaves, of the proportion of leaf area damaged; and 3) the weighted average herbivory severity, that is, total damaged area for all the sampled leaves divided by the total leaf area.

We analyzed each of the three indicators separately, with leaf size In-transformed and herbivore severity logit transformed. Each analysis involved two alternative models: 1) the herbivory index was assumed to monotonically decrease or increase with leaf size, and leaf size was treated as a fixed factor; and 2) a unimodal relationship was expected between leaf size and herbivory index, therefore, leaf size and the quadratic term for leaf size were treated as fixed factors. The better fitting model was selected based on Akaike information criterion (function 'anova' in package 'stats').

A generalized linear mixed model was introduced to test the effect of leaf size on herbivory, for each species. First, we analyzed the effect of leaf size on the probability of leaves being damaged or not damaged by herbivores (i.e., herbivory frequency) with a binomial error distribution (function 'glmer' in package "Ime4'). For the damaged leaves, a linear mixed model was introduced to analyze the effect of leaf size on the proportion of leaf area damaged (i.e., herbivory severity) that was In-transformed to 
reduce skewness (function 'Imer' in package "Ime4'). The same two candidate models (i.e. with and without the quadratic term for leaf size being included as a fixed factor) were used to analyze the leaf size effect, and the better fitting model was selected by using the Akaike information criterion. In all the models, the individual seedling ID nested in plot ID was treated as a random effect.

\section{Results}

\section{General patterns of leaf size and herbivory}

The 174 seedlings from the 30 plots selected in September belonged to 30 species. In total, 2595 leaves were investigated, of which $54.5 \%$ were damaged by herbivores. Both leaf size and severity of herbivory differed greatly among leaves, ranging from $0.02 \mathrm{~cm}^{2}$ to $105.20 \mathrm{~cm}^{2}$ and $0-98 \%$, respectively.

In the 45 plots selected in December, there were 248 seedlings belonging to 37 species. In total, 3159 leaves were investigated, with the herbivory frequency of $58.9 \%$. The leaf size and herbivory severity ranged from $0.06 \mathrm{~cm}^{2}$ to $88.13 \mathrm{~cm}^{2}$, and $0-80 \%$, respectively.

\section{Effects of leaf size on herbivory across species}

In the 30 species surveyed in September, the average leaf size ranged from $0.83 \mathrm{~cm}^{2}$ to $42.30 \mathrm{~cm}^{2}$, the herbivory frequency ranged from 0 to $94.7 \%$, the arithmetic average herbivory severity ranged from 0 to $36 \%$, and the weighted average herbivory severity ranged from 0 to $29 \%$. Leaf size was positively correlated with herbivory frequency (Phylogenetic generalized least squares regression, $t=3.04, p=$ $0.005)$, arithmetic average herbivory severity $(t=2.75, p=0.010)$, and weighted average herbivory severity $(t=2.74, p=0.011)$, indicating that species with larger leaves were more likely to be consumed by herbivores, and were consumed more heavily (Fig. 1).

Among the 37 species surveyed in December, both leaf size and herbivory varied greatly, with the ranges of the parameters being $1.59-35.08 \mathrm{~cm}^{2}$ for leaf size, $0-100 \%$ for herbivory frequency, $0-40.4 \%$ for the arithmetic average herbivory severity, and $0-38.8 \%$ for weighted average herbivory severity. Leaf size exhibited no effect on herbivory frequency, whereas for herbivory severity, a negative quadratic term for leaf size effect was detected in the expressions for both the arithmetic average herbivory severity $(t=$ $-2.86, p=0.008)$ and weighted average herbivory severity $(t=-2.14, p=0.042)$, revealing a hump-shaped pattern which indicates that species producing medium-sized leaves were consumed more heavily than species with both large and small leaves (Fig. 1).

\section{Effects of leaf size on herbivory within species}

Of the 30 species surveyed in September, four species showed a positive correlation between leaf size and herbivory frequency (i.e., larger leaves were more likely to be consumed), one species showed negative correlation (i.e., smaller leaves were more likely to be consumed), three species showed a humpshaped pattern (i.e., medium-sized leaves were more likely to be consumed than both small and large leaves), one species showed a U-shaped pattern (i.e., medium-sized leaves were less likely to be 
consumed than both small and large leaves), and the other 21 species exhibited no apparent patterns (Table 1). For herbivory severity, four species showed a negative correlation between leaf size and the proportion of leaf area consumed by herbivores (i.e., smaller leaves were consumed more heavily), one species showed a U-shaped pattern, and the other 25 species exhibited no apparent patterns (Table 1). 
Table 1

The effects of leaf size on herbivory frequency and severity for each of the 30 species in September. The mean leaf size ( $\mathrm{cm}^{2}$, mean $\pm S D$ ) and sample size (the number in parentheses) are shown in the table. \#: The statistical analysis is based on the generalized linear mixed model with a binomial error distribution; *: The statistical analysis is based on the linear mixed model with gaussian distribution.

\begin{tabular}{|c|c|c|c|c|}
\hline \multirow[t]{2}{*}{ Species } & \multicolumn{2}{|c|}{ Herbivory frequency } & \multicolumn{2}{|l|}{ Herbivory severity } \\
\hline & Leaf size & \# & Leaf size & * \\
\hline Rhododendron leptothrium & $0.83 \pm 0.89(23)$ & - & $0.85 \pm 0.68(7)$ & - \\
\hline Viburnum erubescens & $0.92 \pm 0.65(30)$ & $\nearrow$ & $1.21 \pm 0.73(13)$ & - \\
\hline Schefflera shweliensis & $1.31 \pm 0.99(8)$ & - & $2.04(1)$ & \\
\hline Ligustrum delavayanum & $1.74 \pm 0.99(26)$ & - & $1.64 \pm 0.86(4)$ & - \\
\hline Acanthopanax evodiaefolius & $3.11 \pm 0.21(3)$ & & & \\
\hline Ilex corallina & $3.82 \pm 2.65(486)$ & - & $4.03 \pm 2.65(175)$ & - \\
\hline Viburnum chingii & $4.5 \pm 2.18(43)$ & $\searrow$ & $3.15 \pm 2.35(12)$ & - \\
\hline Meliosma kirkii & $6.06 \pm 4.49(7)$ & - & $8.12(1)$ & \\
\hline Lithocarpus hancei & $6.89 \pm 2.49(14)$ & - & $7.05 \pm 2.51(13)$ & - \\
\hline Schima noronhae & $7.16 \pm 6.8(72)$ & $\nearrow$ & $9.04 \pm 7.41(38)$ & - \\
\hline Ilex szechwanensis & $7.63 \pm 4.76(42)$ & $u$ & $6.53 \pm 5.36(16)$ & - \\
\hline Neolitsea polycarpa & $8.61 \pm 4.88(110)$ & - & $8.47 \pm 4.94(57)$ & - \\
\hline Litsea cubeba & $9.17 \pm 4.85(26)$ & - & $11.25 \pm 5.01(11)$ & - \\
\hline Eriobotrya bengalensis & $9.3 \pm 7.11(14)$ & - & $10.98 \pm 7.05(11)$ & - \\
\hline Styrax perkinsiae & $9.83 \pm 8.67(278)$ & $\nearrow$ & $11.45 \pm 9.56(161)$ & - \\
\hline Litsea elongata & $9.91 \pm 4.71(230)$ & - & $10.02 \pm 4.72(178)$ & $\searrow$ \\
\hline Mahonia bracteolata & $10.46 \pm 6.23(30)$ & $\cap$ & $11.63 \pm 5.32(18)$ & - \\
\hline Laurocerasus undulata & $10.69 \pm 8.44(15)$ & - & $11.21 \pm 8.17(10)$ & - \\
\hline Lindera thomsonii & $11.51 \pm 6.65(12)$ & - & $13.73 \pm 6.61(3)$ & \\
\hline Symplocos poilanei & $12.19 \pm 6.35(30)$ & $\nearrow$ & $14.65 \pm 6.16(16)$ & - \\
\hline Michelia floribunda & $12.68 \pm 7.50(19)$ & - & $12.68 \pm 7.71(18)$ & - \\
\hline Daphne papyracea & $14.60 \pm 6.86(81)$ & - & $15.28 \pm 7.12(48)$ & - \\
\hline
\end{tabular}

$\nearrow$ : positive linear correlation; \: negative linear correlation; $U$ : U-shaped; $\cap$ : hump-shaped; $-:$ means no correlation; and blank: means no analyses were conducted because the sample size was less than 5. 


\begin{tabular}{|c|c|c|c|c|}
\hline \multirow[t]{2}{*}{ Species } & \multicolumn{2}{|l|}{ Herbivory frequency } & \multicolumn{2}{|l|}{ Herbivory severity } \\
\hline & Leaf size & \# & Leaf size & * \\
\hline Symplocos ramosissima & $14.79 \pm 9.11(390)$ & - & $14.37 \pm 9.33(263)$ & $\searrow$ \\
\hline Symplocos dryophila & $15.05 \pm 6.84(61)$ & - & $14.67 \pm 6.27(41)$ & $\searrow$ \\
\hline Stewartia pteropetiolata & $16.04 \pm 12.27(234)$ & $\cap$ & $17.78 \pm 11.83(120)$ & - \\
\hline Viburnum cylindricum & $17.20 \pm 7.72(32)$ & $\cap$ & $18.22 \pm 7.11(16)$ & - \\
\hline Machilus gamblei & $20.16 \pm 13.99(131)$ & - & $21.51 \pm 15.29(84)$ & $\searrow$ \\
\hline Manglietia insignis & $23.01 \pm 16.41(65)$ & - & $21.21 \pm 16.37(21)$ & - \\
\hline Aucuba himalaica & $25.03 \pm 18.52(53)$ & - & $24.75 \pm 18.02(41)$ & - \\
\hline Illicium macranthum & $42.3 \pm 14.43(30)$ & - & $41.70 \pm 8.31(17)$ & $u$ \\
\hline \multicolumn{5}{|c|}{$\begin{array}{l}\text { ᄀ: positive linear correlation; \: negative linear correlation; } \cup \text { : U-shaped; } \cap \text { : hump-shaped; -: means } \\
\text { no correlation; and blank: means no analyses were conducted because the sample size was less than } \\
5 \text {. }\end{array}$} \\
\hline
\end{tabular}

Of the 37 species surveyed in December, four species showed a positive correlation between leaf size and herbivory frequency, one species showed negative correlation, one species exhibited a hump-shaped pattern (i.e., medium-sized leaves were more likely to be consumed than both small and large leaves), one species exhibited a U-shaped pattern (i.e., medium-sized leaves were less likely to be consumed than both small and large leaves), and the other 30 species exhibited no apparent patterns (Table 1). For herbivory severity, seven species exhibited a negative correlation, while the other 30 species displayed no apparent patterns (Table 2). 
Table 2

The effects of leaf size on herbivory frequency and severity for each of the 37 species in December. Details of the explanations of the values in the table, please see the caption of Table 1.

\begin{tabular}{|lllll|}
\hline Species & Leaf size & \# & Leaf size & $*$ \\
\hline Ligustrum delavayanum & $1.09 \pm 0.94(56)$ & - & $1.01 \pm 0.87(15)$ & - \\
\hline Rhododendron leptothrium & $1.11 \pm 0.86(23)$ & $\cap$ & $1.86 \pm 0.63(9)$ & - \\
\hline Eurya jintungensis & $2.04 \pm 1.24(15)$ & - & $2.12 \pm 1.05(11)$ & - \\
\hline Rhododendron decorum & $2.67 \pm 1.08(8)$ & & & \\
\hline Viburnum chingii & $2.95 \pm 2.24(46)$ & - & $2.19 \pm 1.66(13)$ & - \\
\hline Zanthoxylum esquirolii & $3.20 \pm 1.52(30)$ & - & $2.93 \pm 1.72(13)$ & - \\
\hline Ilex coralline & $3.35 \pm 2.26(315)$ & $\nearrow$ & $3.49 \pm 2.30(119)$ & $\searrow$ \\
\hline Ilex szechwanensis & $3.54 \pm 2.88(86)$ & - & $3.33 \pm 2.92(36)$ & $\searrow$ \\
\hline Ilex gingtungensis & $4.26 \pm 2.89(15)$ & - & $4.65 \pm 2.73(11)$ & - \\
\hline Ilex gingtungensis & $4.47 \pm 1.13(4)$ & & $4.47 \pm 1.13(4)$ & \\
\hline Schefflera shweliensis & $4.62 \pm 3.03(47)$ & - & $4.95 \pm 3.10(15)$ & - \\
\hline Prunus tomentosa & $6.23 \pm 1.46(3)$ & & $7.23 \pm 0.67(2)$ & \\
\hline Litsea elongate & $6.57 \pm 2.48(16)$ & - & $7.34 \pm 2.36(6)$ & - \\
\hline Litsea cubeba & $6.96 \pm 6.16(7)$ & - & $9.12 \pm 6.76(5)$ & \\
\hline Laurocerasus undulate & $7.25 \pm 2.73(10)$ & - & $7.05 \pm 2.97(9)$ & - \\
\hline Ilex manneiensis & $7.7 \pm 4.21(11)$ & - & $8.63 \pm 4.44(6)$ & - \\
\hline Mahonia bracteolate & $8.6 \pm 5.28(30)$ & - & $10.01 \pm 7.34(11)$ & - \\
\hline Prunus cerasoides & $8.66 \pm 3.55(4)$ & & $8.66 \pm 4.10(4)$ & \\
\hline Eriobotrya bengalensis & $8.79 \pm 4.93(21)$ & - & $8.29 \pm 4.62(20)$ & - \\
\hline Neolitsea polycarpa & $9.85 \pm 6.18(469)$ & $\nearrow$ & $10.90 \pm 6.81(288)$ & - \\
\hline Schima noronhae & $10.30 \pm 7.05(36)$ & - & $11.55 \pm 6.52(20)$ & - \\
\hline Lithocarpus hancei & $10.36 \pm 4.09(21)$ & $\searrow$ & $9.90 \pm 4.20(18)$ & - \\
\hline Viburnum cylindricum & $10.50 \pm 7.17(22)$ & - & $12.58 \pm 6.98(10)$ & $\searrow$ \\
\hline Machilus gamblei & $12.37 \pm 7.05(397)$ & - & $12.86 \pm 7.45(247)$ & $\searrow$ \\
\hline Michelia floribunda & $13.56 \pm 7.15(16)$ & - & $13.56 \pm 7.15(16)$ & - \\
\hline Symplocos ramosissima & $13.62 \pm 8.83(422)$ & - & $14.14 \pm 9.13(315)$ & $\searrow$ \\
\hline
\end{tabular}




\begin{tabular}{|lllll|}
\hline Species & Leaf size & \# & Leaf size & * \\
\hline Symplocos anomala & $14.67 \pm 4.74(33)$ & - & $13.81 \pm 5.73(18)$ & - \\
\hline Daphne papyracea & $14.80 \pm 6.65(103)$ & - & $14.62 \pm 6.38(85)$ & $\searrow$ \\
Cyclobalanopsis stewardiana & $14.93 \pm 4.84(30)$ & - & $15.25 \pm 4.89(24)$ & - \\
Stewartia pteropetiolata & $15.25 \pm 12.38(381)$ & $\nearrow$ & $17.51 \pm 12.78(218)$ & $\searrow$ \\
Symplocos dryophila & $16.25 \pm 7.44(96)$ & - & $17.35 \pm 8.17(61)$ & - \\
Ardisia crenata & $17.05 \pm 10.77(26)$ & - & $18.26 \pm 10.11(13)$ & - \\
Lindera thomsonii & $20.45 \pm 3.55(29)$ & - & $21.83 \pm 3.39(11)$ & - \\
\hline Symplocos poilanei & $21.42 \pm 12.63(90)$ & $\nearrow$ & $23.35 \pm 12.39(77)$ & - \\
\hline Skimmia arborescens & $23.08 \pm 11.06(30)$ & $\cup$ & $20.64 \pm 12.32(16)$ & - \\
Illicium macranthum & $33.09 \pm 13.26(58)$ & - & $35.52 \pm 13.13(32)$ & - \\
\hline Manglietia insignis & $35.08 \pm 20.36(153)$ & - & $35.84 \pm 21.41(81)$ & - \\
\hline
\end{tabular}

\section{Discussion}

Overall, our results provide strong evidence that the relationship between leaf size and insect herbivory does not follow a constant rule across seasons; a pattern of monotonic increase occurred in September, while a hump-shaped pattern occurred in December. More complex patterns was detected among species, showing a variety of patterns including hump-shaped and U-shaped patterns, and patterns with monotonic decrease and increase, although most species showed a leaf-size-independent pattern.

As discussed previously, various mechanisms determine both the positive and negative effects of leaf size on herbivory (Feeny 1976; Bogacheva 1994; Moles and Westoby 2000; Low et al. 2009; Moreira et al. 2016). In this study, the leaves differed greatly not only in leaf size, but also in other traits such as specific leaf area, and carbon and nitrogen content (unpublished data), all of which may affect the foraging preferences of insect herbivores (Ackerly et al. 2002; Cardenas et al. 2014; Zava and Cianciaruso 2014), thus influencing the effects of leaf size on herbivory. Despite potential interference from other factors, our results showed a clear positive relationship between herbivory and leaf size in September; larger leaves were more likely to be damaged by insects and suffered heavier damage than smaller ones, indicating that the positive effects of leaf size may overcome the negative effects. However, a humpshaped pattern was found in December, that is, the consumption of medium-sized leaves was higher than that of both large and small ones. In our study area, the temperature was much lower in December than in September $\left(7.2^{\circ} \mathrm{C}\right.$ vs. $\left.15.3^{\circ} \mathrm{C}\right)$. In general, ectothermic insect herbivores are sensitive to temperature reduction, and often show a decrease in abundance, activity, and metabolic intensity, as well as reduced energy requirements at lower temperatures (Sinclair et al. 2003; Deutsch et al. 2008; Rho and Lee 2017). In such situations, based on the predator satiation hypothesis (Janzen 1971), large-sized leaves may 
show a smaller proportion of insect damage than medium-sized leaves, as the absolute nutrient content of large leaves far exceeds the energy requirement of the insect herbivores.

Our results showed that the leaf size preference varied considerably among species in both seasons, indicating that the effect of leaf size on insect herbivory was species-specific. Similar interspecific variation has been reported in other species interactions such as seed dispersal and predation by small mammals (Feng et al. 2021; Thein et al. 2021). The species measured in our study differed greatly in the mean value $\left(0.83-42.30 \mathrm{~cm}^{2}\right)$ and range $\left(0.46-104.69 \mathrm{~cm}^{2}\right)$ of leaf size, and the ratio of maximum to minimum leaf size (2-617-fold). However, most of them did not show clear effects of leaf size on herbivory within the species. Here, we propose several possible explanations: 1) the limited range of leaf size within a single species may not be sufficient to exhibit a clear effect of size on herbivory. 2) Plant leaves usually possess physical and chemical defense traits in varying degrees (Moles et al. 2013; Cardenas et al. 2014; Zava and Cianciaruso 2014; Zhao et al. 2021), which may influence the effects of leaf size on herbivory; for example, insect herbivores may reject a leaf regardless of whether it is large or small, if the leaf contains a large amount of highly toxic chemicals. 3) Leaves often differ in nutrient content among species (Kattge et al. 2011; Cardenas et al. 2014), and the nutrient content of a leaf may also introduce biases on the effects of leaf size on herbivory. 4) Many insects are specialist herbivores (Ali and Agrawal 2012), and different species of herbivores may show different preferences for leaf size (Bogacheva 1994). Furthermore, the neighbor effect also affects insect-leaf interactions; for example, whether a given species of plant would be consumed by an insect herbivore can be influenced by the presence of neighboring plants (Baraza et al. 2006; Hahn and Orrock 2016; Moreira et al. 2017). The neighbor effect often manifests as different or even opposite patterns, depending on the species present in the neighborhood (Wang et al. 2010, Castagneyrol et al. 2018, Wang 2020). In our study, the sample plots were randomly selected; therefore, for any given species of leaves, the neighbor effect may differ among plots because of the differences in species composition among the plots. Such variations in the neighbor effect may dilute the effect of leaf size on the preference of insect herbivores for a specific species of leaves.

The effect of leaf size on insect herbivory varied across seasons and among plant species, In conclusion, the effects of leaf size on the foraging preferences of insect herbivores may be contingent on both external (e.g., temperature) and intrinsic (e.g., other leaf traits) factors, indicating that a one-off survey with a few species may bias our understanding on the overall pattern of the effect of leaf size on herbivory. Similar variations may also exist in other systems (e.g., pollination, frugivory, seed predation, etc.), and should be considered in future studies on biotic interactions.

\section{Declarations}

\section{Acknowledgments}

We thank Ailaoshan Station of Subtropical Forest Ecosystem Studies for logistical support in the field work. This study was funded by the National Natural Science Foundation of China (31971444 and 
32171533).

\section{Funding}

This study was supported by the National Natural Science Foundation of China (31971444 and 32171533).

\section{Data Availability}

The datasets generated and analyzed duringthe current study are available from the corresponding author onreasonable request.

Code availability

Not applicable.

Animal Research (Ethics)

Not applicable.

Consent to Participate (Ethics)

Not applicable.

Consent to Publish (Ethics)

Not applicable.

Plant Reproducibility

Not applicable.

\section{Clinical Trials Registration}

Not applicable.

Conflict of Interest

The authors have no relevant financial or non-financial interests to disclose.

\section{Author Contributions:}

Bo Wang conceived and designed the experiments. Xiang Zhang and Li Feng performed the experiments. Bo Wang, Xiang Zhang and Li Feng analyzed the data and wrote first draft of the manuscript, and all authors commented on previous versions of the manuscript.All authors read and approved the final manuscript. 


\section{References}

1. Ackerly D, Knight C, Weiss S, Barton K, Starmer K (2002) Leaf size, specific leaf area and microhabitat distribution of chaparral woody plants: contrasting patterns in species level and community level analyses. Oecologia 130:449-457

2. Aide TM (1993) Patterns of leaf development and herbivory in a tropical understory community. Ecology 74:455-466

3. Ali JG, Agrawal AA (2012) Specialist versus generalist insect herbivores and plant defense. Trends Plant Sci 17:293-302

4. Baraza E, Zamora R, Hodar JA (2006) Conditional outcomes in plant-herbivore interactions: neighbours matter. Oikos 113:148-156

5. Barton KE, Hanley ME (2013) Seedling-herbivore interactions: insights into plant defence and regeneration patterns. Ann Bot 112:643-650

6. Bogacheva IA (1994) Leaf size selection by insects: a phenomenon created by random sampling. Oikos69:119-124

7. Bogacheva IA (2002) Size-dependent selective leaf damage by insects and some methodological implications of this phenomenon. Russ JEcol 33:423-428

8. Brown VK, Lawton JH (1991) Herbivory and the evolution of leaf size and shape. Philos Trans R Soc B 333:265-272

9. Cardenas RE, Valencia R, Kraft NJ, Argoti A, Dangles O (2014) Plant traits predict inter-and intraspecific variation in susceptibility to herbivory in a hyperdiverseNeotropical rain forest tree community. J Ecol 102:939-952

10. Castagneyrol B, Jactel H, Moreira X (2018) Anti-herbivore defences and insect herbivory: interactive effects of drought and tree neighbours. J Ecol 106:2043-2057

11. Coley P. (1980)Effects of leaf age and plant life history patterns on herbivory. Nature 284:545-546

12. Deutsch CA, Tewksbury JJ, Huey RB, Sheldon KS, Ghalambor CK, Haak DC, Martin PR (2008) Impacts of climate warming on terrestrial ectotherms across latitude. ProcNatlAcadSci USA 105:6668-6672

13. Diaz S, Kattge J, Cornelissen JH, Wright IJ, Lavorel S, Dray S, et al (2016) The global spectrum of plant form and function. Nature 529:167-171

14. Eichhorn MP, Nilus R, Compton SG, Hartley SE, Burslem DF (2010) Herbivory of tropical rain forest tree seedlings correlates with future mortality. Ecology 91:1092-1101

15. Eisenring M, Unsicker SB, Lindroth RL (2021) Spatial, genetic and biotic factors shape within-crown leaf trait variation and herbivore performance in a foundation tree species. FunctEcol 35:54-66

16. Elliott S, Irwin R (2009) Effect of flowering plant density on pollinator visitation, pollen receipt and seed production in Delphinium barbeyi (Ranunculaceae). Am J Bot 96:912-9 
17. Falster DS,Westoby M (2003) Leaf size and angle vary widely across species: what consequences for light interception? New Phytol 158:509-525

18. Feeny P (1976) Plant apparency and chemical defense.In: Wallace JW,Mansell RL(eds) Recent advances in phytochemistry. Plenum Press, New York,pp 1-40

19. Feng L, Chen S, Wang B (2021) Fine-scale spatiotemporal variation in seed-rodent interactions: a potential contribution to species coexistence. For Ecol Manage 498:119566

20. Hahn PG, Orrock JL (2016) Neighbor palatability generates associational effects by altering herbivore foraging behavior. Ecology 97:2103-2111.

21. Hu W, Lu Z, Meng F, Li X, Cong R, Ren T, Sharkey TD, Lu J (2020) The reduction in leaf area precedes that in photosynthesis under potassium deficiency: the importance of leaf anatomy. New Phytol 227:1749-1763

22. Hulshof CM, Swenson NG (2010) Variation in leaf functional trait values within and across individuals and species: an example from a Costa Rican dry forest. FunctEcol 24:217-223

23. Jactel H, Moreira X, Castagneyrol B (2021) Tree diversity and forest resistance to insect pests: patterns, mechanisms, and prospects. Annu Rev Entomol 66:277-296

24. Janzen DH (1971) Seed predation by animals.Annu Rev EcolSyst 2:465-492.

25. Kattge J, Diaz S, Lavorel S, Prentice IC, Leadley P, Bönisch G, et al (2011) TRY-a global database of plant traits. Global change biol 17:2905-2935

26. Leigh A, Sevanto S, Close JD, Nicotra AB (2017) The influence of leaf size and shape on leaf thermal dynamics: does theory hold up under natural conditions? Plant Cell Environ 40:237-248

27. Li W, Chen Y, Shen Y, Lu Y, Yu S (2021) Plant trait differences and soil moisture jointly affect insect herbivory on seedling young leaves in a subtropical forest. For Ecol Manage 482:118878

28. Low C, Wood SN, Nisbet RM (2009) The effects of group size, leaf size, and density on the performance of a leaf-mining moth. J AnimEcol 78:152-160

29. McDonald PG, Fonseca CR, Overton JM,Westoby M (2003) Leaf-size divergence along rainfall and soil-nutrient gradients: is the method of size reduction common among clades? FunctEcol 17:50-57

30. Moles AT, Westoby M (2000) Do small leaves expand faster than large leaves, and do shorter expansion times reduce herbivore damage? Oikos 90:517-524

31. Moles AT, Peco B, Wallis IR, Foley WJ, Poore AG, Seabloom EW, et al (2013) Correlations between physical and chemical defences in plants: tradeoffs, syndromes, or just many different ways to skin a herbivorous cat? New Phytol 198:252-263

32. Moreira X, Glauser G,Abdala-Roberts L (2017) Interactive effects of plant neighbourhood and ontogeny on insect herbivory and plant defensive traits. SciRep 7:4047

33. Moreira LF, Teixeira NC, Santos NA, Valim JOS, Maurício RM, Guedes RNC, et al (2016) Diamondback moth performance and preference for leaves of Brassica oleracea of different ages and strata. $\mathrm{J}$ ApplEntomol 140:627-635 
34. Poorter L, Rozendaal DM (2008) Leaf size and leaf display of thirty-eight tropical tree species. Oecologia 158:35-46

35. Potter KA, Bronstein J,Davidowitz G (2012) Choice of oviposition sites by Manducasexta and its consequences for egg and larval performance. EntomolExpAppl 144:286-293

36. Rho MS, Lee KP (2017) Temperature-driven plasticity in nutrient use and preference in an ectotherm. Oecologia 185:401-413

37. Schowalter TD, Hargrove W, CrossleyJr DA (1986) Herbivory in forested ecosystems. AnnuRev Entomol 31:177-196

38. Sinclair BJ, Vernon P, Klok CJ, Chown SL (2003) Insects at low temperatures: an ecological perspective. Trends EcolEvol 18:257-262

39. Thein MM, Wu LM, Corlett RT, Quan RC,Wang B (2021) Changes in seed predation along a 2300-m elevational gradient on a tropical mountain in Myanmar: a standardized test with 32 non-native plant species. Ecography 44:602-611

40. Wang L, Wang D, Bai Y, Huang Y, Fan M, Liu J,Li Y (2010) Spatially complex neighboring relationships among grassland plant species as an effective mechanism of defense against herbivory. Oecologia 164:193-200

41. Wang B (2020)Neighbour effects do not always show consistent patterns, contrast of seed trait matters: evidence from a seed-rodent mutualism study. BehavEcolSociobiol 74:119

42. Wright IJ, Dong N, Maire V, Prentice IC, Westoby M, Diaz S, et al (2017) Global climatic drivers of leaf size. Science 357:917-921

43. Yan C, Zhang Z (2014) Specific non-monotonous interactions increase stability of ecological networks. Proc R Soc B 281:20132797

44. Yates MJ, Anthony-Verboom G, Rebelo AG, Cramer MD (2010) Ecophysiological significance of leaf size variation in Proteaceae from the Cape Floristic Region. FunctEcol 24:485-492

45. Zhang S, Zhang Y,Ma K (2016) Latitudinal variation in herbivory: hemispheric asymmetries and the role of climatic drivers. J Ecol 104:1089-1095

46. Zhang Z, Yan C, Zhang H (2021) Mutualism between antagonists: its ecological and evolutionary implications. IntegrZool 16:84-96

47. Zhao J, Segar ST, McKey D, Chen J (2021) Macroevolution of defense syndromes in Ficus (Moraceae). EcolMonogr 91:e01428

48. Zava PC, Cianciaruso MV (2014) Can we use plant traits and soil characteristics to predict leaf damage in savanna woody species? Plant Ecol 215:625-637

\section{Figures}

\section{Figure 1}


Effects of leaf size on herbivory frequency $(a, b)$, herbivory severity that derived from the arithmetic average formula $(c, d)$ and $(e, f)$ herbivory severity that derived from the weighted average formula. The regression lines were based on the phylogenetic generalized least squares regression (PGLS). 